\title{
NUMERICAL ANALYSIS OF PROPELLANT BEHAVIOR IN TANKS: FROM SMALL-SCALE TO INDUSTRIAL MODELS
}

\author{
A. Dufour \\ EADS ASTRIUM Space Transportation \\ BP3002, Route de Verneuil 66, Les Mureaux 78133, France
}

This document presents some analysis performed in the frame of COMPERE program [1]. First, the COMPERE Program is presented: participants and main objectives. Then, the results obtained on small-scale experiment computations are analyzed. The third point deals with the computations of ground tests performed in an industrial environment. Finally, future works on propellant management are discussed.

\section{INTRODUCTION}

When designing liquid launcher stages, it is mandatory to have good knowledge on propellant behavior in the tanks. For cryogenic stages with several coasting phases, arising phenomena become more difficult to assess: microgravity modifies the heat exchange (bulk/wall, bulk/ullage, and ullage/wall) and induces large motions in the tanks. In order to improve the knowledge on such topics, a research program has been launched by two national agencies CNES (France) and DLR (Germany) in cooperation with Ariane industrials (Air Liquide, Astrium), research organizations (ONERA) and academic laboratories (LEGI Grenoble, IMFT Toulouse, ZARM Bremen, Erlangen University, and some others). During the launcher flight, different conditions exist. They have been categorized in four main phases:

(1) propelled phase;

(2) ballistic phase;

(3) preparation of engine restart; and

(4) depressurization.

This is an Open Access article distributed under the terms of the Creative Commons Attribution-Noncommercial License 3.0, which permits unrestricted use, distribution, and reproduction in any noncommercial medium, provided the original work is properly cited. 
These phases deal with different phenomena and external conditions which influence the launcher characteristics. The main issues to be addressed are:

- pressurization budget (inert mass vs. payload, evaporation, condensation, sloshing, etc.);

- piloting (sloshing characterization);

- residuals (stratification of the first boost); and

- additional issues (design oriented).

Other issues address the ballistic phase:

- engine reignition (pressure, temperature, settling, etc.); and

- residuals (temperature gradient, additional boosts).

The COMPERE program has been started in 2000 to support the development of future upper cryogenic stages for Ariane 5 launcher. This objective remains valid and propellant management will be one of the main tasks for the A5ME upper stage. The existing stages like EPC and EPS can also benefit from the program.

Presented here are some works performed by the Astrium Space Transportation aimed at improving the knowledge for the development of tools predicting propellant behavior. It only represents a small part of the works performed in the frame of COMPERE by all program partners. The way to proceed is a stepby-step approach: from small-scale to large-scale, and with several acceleration conditions at microgravity and ground conditions.

\section{PROPELLANT REORIENTATION CASE}

One of the existing problems is how the propellant will behave in the case of thrust decay. It may be applied to first stage separation when the acceleration of the first stage becomes negative and induces the motion of residuals from the lower to the upper bulkhead of the tanks. It is also applicable to the upper stages when the coasting phase starts.

This configuration was studied by ZARM Institute both for cryogenic and non-cryogenic fluids. The thrust decay was simulated by a step reduction of gravity thanks to drop tower experiment. The Astrium Space Transportation performed some computations on this kind of experiments in cooperation with the teams involved in COMPERE.

The initial configuration is liquid settled in the bottom of tank (representative of high acceleration, for instance, boosted phase). When engine switches off, the 
acceleration falls down suddenly to a value close to zero. Consequently, that induces reorientation of the fluid and free surface evolution. The direct result will be a large climbing of fluid along the wall (driven by capillarity phenomena) and oscillations of contact point (fluid/wall) vs. time. It may occur for a stage separation or for an intermediate engine shutdown. In both cases, the knowledge of the fluid position and state is mandatory.

In the experimental setup designed by ZARM Institute [2], the reorientation occurs on the inner wall of an annular cylinder, which is filled by liquid. The outer wall is isolated from fluid wetting during reorientation by adding a sharp edge which keeps the external contact point pinned to it. A heater at the $z$-axis (symmetry axis) provides heat fluxes partially along the walls and then allows nonisothermal experiment: liquid and wall are not at the same temperature. Figure 1 shows schematic view of the test device. The geometry and input data have been provided by ZARM Institute, as well as experimental results (temperature probes, heater calibration video).

For all the computations performed by Astrium, the FLOW3D ${ }^{\circledR}$ CFD tool was used. It is a well-known computer code developed by Flow Science and widely used for simulating sloshing and other fluid phenomena. The main numerical assumptions adopted in the code are the second-order computations time and space. The flow is supposed to be laminar (no turbulence model used). The antisymmetric mesh well adapted to the annular cylinder geometry is defined here. The computational domain is an

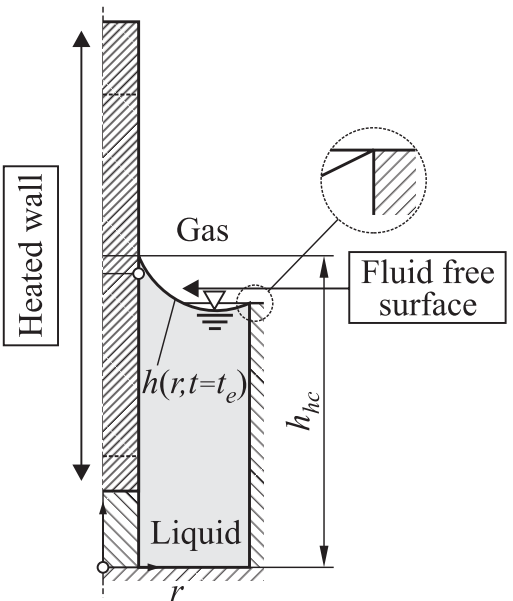

Figure 1 Nonisothermal reorientation experiment angular sector (two cells) with boundary conditions at each face including a heater. A simulation of heat conduction in the heater coupled with fluid motion and fluid/wall heat exchange. It is important, because it may allow, in the future, performing such computations for a "real" tank (from external wall boundary conditions to bulk). Here, a regular grid is used; the cells are close to squares in the symmetry plane in order to limit mesh discrepancies which may degrade the results.

The available experimental data are the fluid level near the wall as well as fluid temperatures close to the wall. The time histories of the contact angle and free surface shapes have been obtained by video image analysis.

Two meshes have been used for the computations: one "coarse" (mesh 1: 150 $\times 470)$ and one "fine" (mesh 2: $225 \times 705$ ), in order to verify the convergence of 


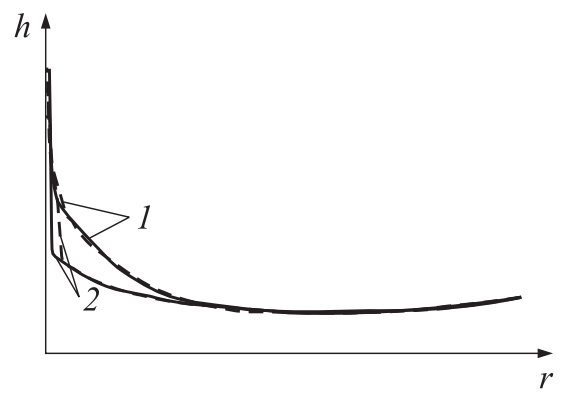

Figure 2 Mesh influence (solid curves - mesh 1 and dashed curves mesh 2) on free surface shape: $1-t$ $=0.43 \mathrm{~s}$ and $2-t=4.70 \mathrm{~s}$

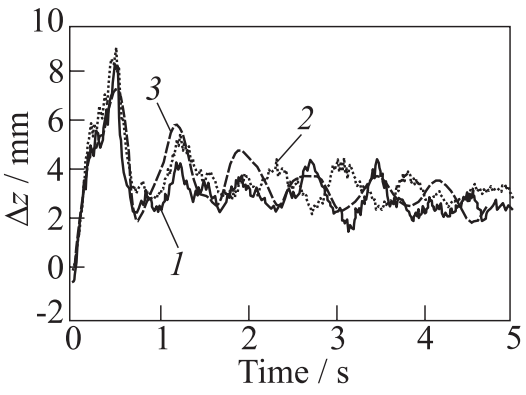

Figure 3 Comparison of fluid height at walls: 1 - mesh 1 (FLOW3D); $2-$ mesh 2 (FLOW3D); and 3 - experiment

the computations. One can see in Fig. 2 the comparison between the shapes of the free surface for two different times.

At the end of reorientation $(t=4.7 \mathrm{~s})$, the shape is very coherent. Only a small discrepancy exists which is limited to the part close to the wall.

Then, the results of calculations were compared with the experimental results. Figure 3 shows the fluid height at the wall as a function of time. The first two oscillations are well captured by the code irrespectively of the mesh used. Thereafter, the predicted frequencies of the phenomenon differ from those in the experiment. The average value of the predicted fluid height is correct, but there are opposite oscillations, mainly, for the fine mesh.

In the frame of nonisothermal reorientation, several boundary conditions have been tested (heat fluxes). Two studies were performed: one with FLOW3D ${ }^{\circledR}$ for commercial code benchmarking (Astrium computations with different heat fluxes; one value is presented here, see also [2]) and another with the academic tool in order to identify the dependency of the contact angle on nonisothermal conditions not provided by the commercial code.

After the reorientation phase, for preparing engine reignition, a settling phase starts. This is the purpose of the following experiment designed and computed in the scope of the COMPERE program [3].

\section{GEYSERING}

In the previous section, engine shutdown has been discussed. After the shutdown, the propellant is located in the upper part of the tank. In order to have the propellant for chilling down and then ignition, settling will be performed. During this transient phase, the liquid falls along the wall and can generate a geyser. 
An experiment has been performed by LEGI [3] on the ground with substitute fluids (Fig. 4). It was designed with a membrane for maintaining the liquid in the upper part of the vessel on the ground. The membrane was then broken by a needle, which directly induced liquid reorientation due to normal gravity on the ground. Experimental data are the coordinate of the fluid position along the wall and the height of the geyser at the axis. These results have been obtained by video analysis. As for the previous case, four meshes were used: $50 \times 100,100 \times 200$, $150 \times 300$, and $200 \times 400$.

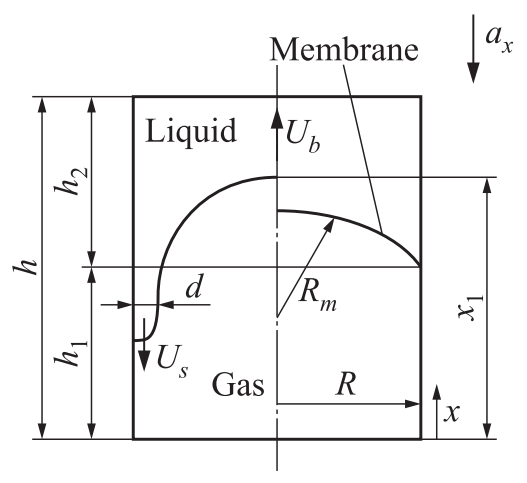

Figure 4 Schematics of LEGI experiment

\subsection{Liquid Film Falling and Bubble Height}

Figure 5 shows the variation of fluid height during falling. The data have been normalized by the total height and characteristic film time. The latter corresponds to the falling time without transient bubble regime $(\sqrt{h / g})$. Due to this definition, the normalized values are higher than 1 . The comparison between the results obtained for the four meshes and experimental data is also made in Fig. 5. Clearly, there is a very small effect of the computational mesh size on the normalized height. As could be expected, the finer the mesh, the better the

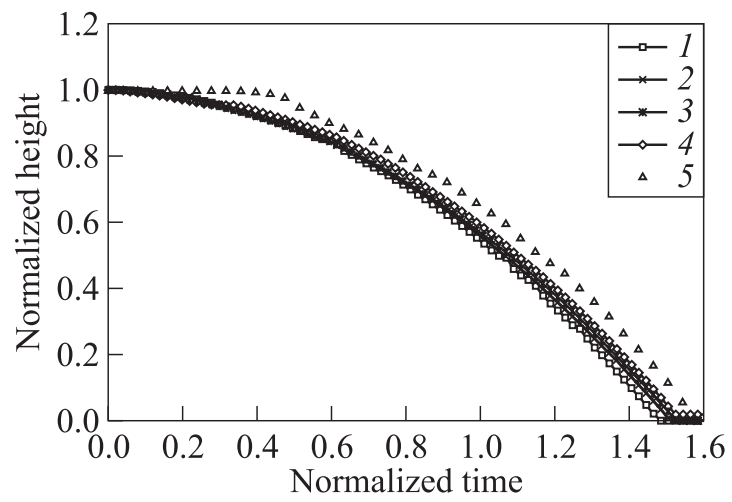

Figure 5 Evolution of falling height vs. time: 1-4 - meshes 1 to 4 (FLOW3D); and 5 - LEGI FC77 experiment 
results. There is a small discrepancy at the beginning of falling, may be due to the experimental device which delays the start of liquid motion. Except for this point, the theoretical and experimental values are in good agreement.

\subsection{Geyser Formation}
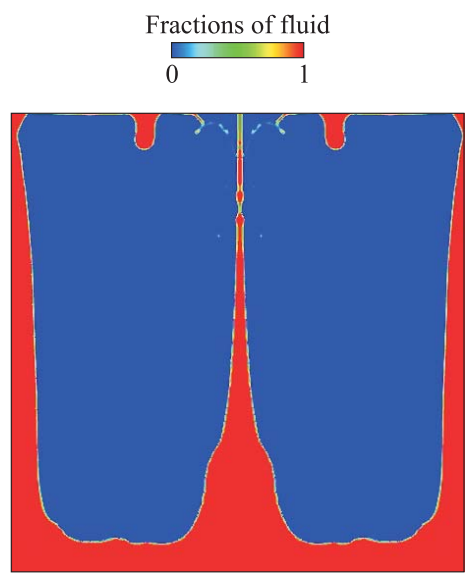

Figure 6 Geyser formation. (Refer Dufour, p. 722.)
After liquid film formation and falling at the vertical wall, the liquid follows the lower vessel wall from each side of the axis. When the flows meet at the axis, a geyser forms. It is well illustrated Fig. 6 extracted from computer simulation. Plotted in this figure, the fraction of fluid: 1.0 stands for pure liquid whereas 0.0 stands for pure gas. One can see here the liquid impact against the top of the vessel. The data of most interest for stage configuration are the geyser occurrence and its related height (if any).

Figure 7 shows the predicted time history of geyser height. The geyser height appeared to be more sensitive to the mesh size as compared to the film falling phenomenon.

Mesh refinement has a strong effect on the geyser growth rate, especially when one switches from the most coarse mesh to the first intermediate mesh.

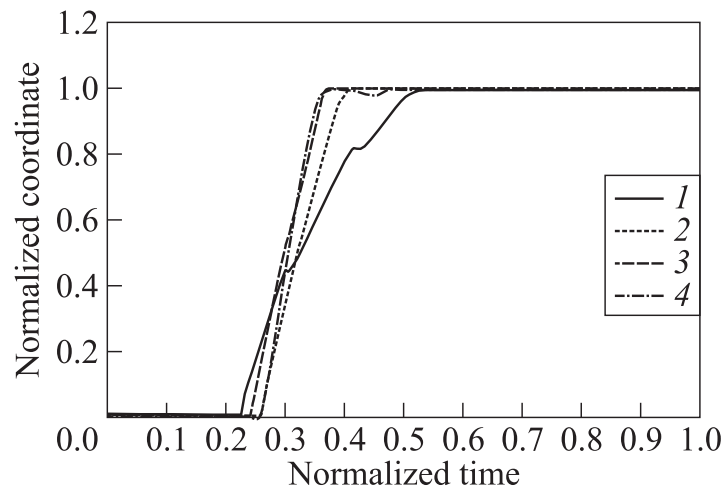

Figure 7 Geyser height vs. time: $1-4-$ meshes 1 to 4 (FLOW3D FC77) 
The time taken for the geyser to hit the vessel upper wall (impact on slope) is also different for different meshes. However, for the three refined meshes, the differences are not that high. Unfortunately, the experimental data are not available for comparison: the experimental geyser was fully three-dimensional (3D) due do parasitic liquid jets acting as dampers of geyser formation.

\section{STRATIFICATION AND PRESSURIZATION}

Another point one has to deal with is the pressure and temperature in the propellant tanks. To perform the first step in the analysis, it was decided to focus on an experiment designed and operated by Air Liquide in the frame of Ariane launcher studies. A tank designed for ground testing and used for both stratification and sloshing tests was considered. The propellant was liquid hydrogen. Several measurements were involved: fluid level, temperature probes for the ullage and the bulk, as well as pressure gauges. Figure 8 shows the geometry of the tank and the mesh used. It is a cylindrical tank ended by two spherical bulkheads.

A lot of potential reference experiments were performed. However, this experiment was chosen because it corresponds to an industrial experiment with relatively "good" scales and with temperature and pressure measurements. The boundary conditions (thermal fluxes, motion) are also relatively well known. All

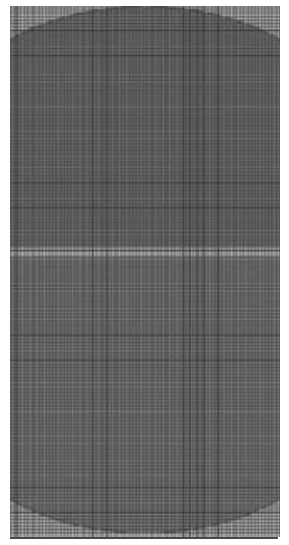

Figure 8 Geometry and mesh of the cylindrical tank these features provide configuration close to the launcher-related architecture and allow direct application of the computational fluid dynamics (CFD) code for improving the methodology of temperature and pressure prediction. As several configurations were tested (self-pressurization, draining, sloshing), it offers a potential to cover a wide range of phenomena encountered during the launcher life.

The first problem decided be addressed was self-pressurization and stratification without draining and sloshing. The reason was to always keep the step-by-step approach.

The computation performed was two-dimensional to be directly comparable in the future studies. The axisymmetric mesh was not used because at the end of the study, a real 3D tank computation with lateral and longitudinal forces inducing sloshing (e.g., flight simulation) is planned.

Here, only the stratification phase is simulated. The power inputs into the tank were set at lateral walls and bulkhead. Some "numerical probes" for pres- 


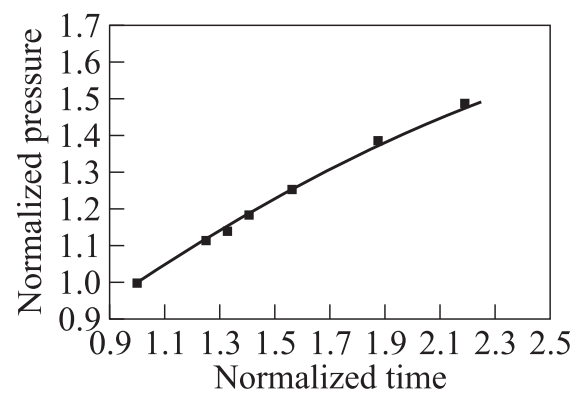

Figure 9 Ullage pressure comparison: curve - computation and signs - experiment sure and temperature measurements were also implemented corresponding to physical measurements. The results are presented in the reduced form: the time is normalized by the time of selfpressurization start and pressure - by the corresponding initial value.

One can see a good agreement of predicted and measured pressure histories during tank self-pressurization for the ground conditions (Fig. 9). The prediction has been obtained by the computation taking into account bulk and ullage stratification as FLOW3D version 9.3 allows.

Figure 10 shows the predicted and ullage temperatures. As can be seen, there is a small asymmetry in the temperature field. Detailed analysis of the velocity field showed that it was not symmetric which influenced the temperature field. Therefore, it was decided to refine the mesh in order to have a more accurate resolution of the flow field. The coarse mesh size was increased from $75 \times 150$ to $100 \times 200$.
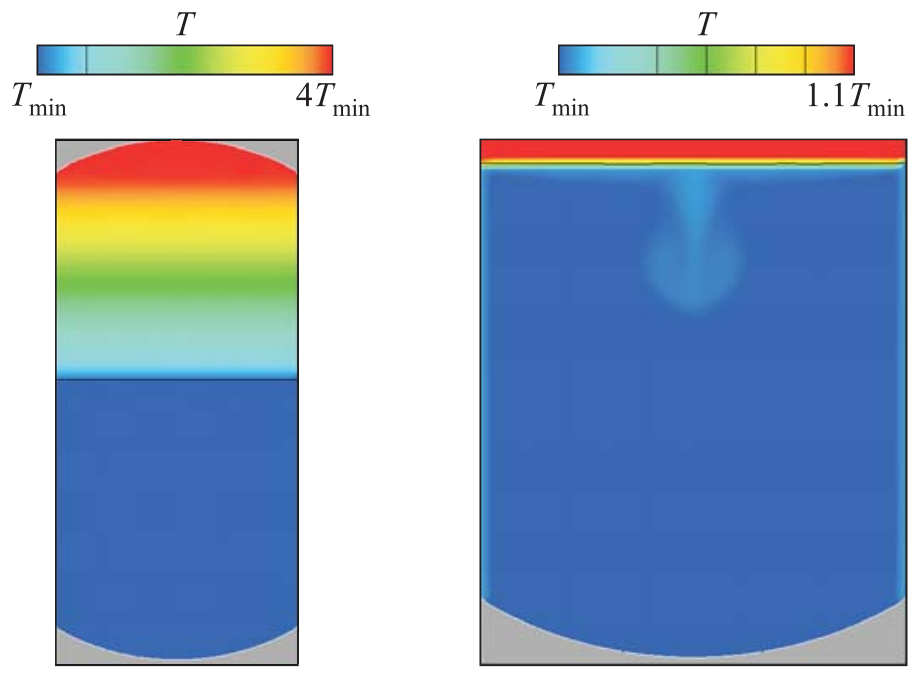

Figure 10 Temperature stratification in the tank (ullage and bulk). (Refer Dufour, p. 724.) 
Figure 11 compares the predicted velocity fields for an intermediate time near the interface which drives the temperature field. One can see that there is some improvement in the symmetry of the flow field. Pressure levels are always well predicted.

Mesh refinement stabilizes the temperature fields and removes the oscillations seen in Fig. 12. In parallel, the increase in temperature becomes smoother. The comparison was made for three different times (50, 100, and $150 \mathrm{~s}$ ).

When comparing the predictions with experimental results, it appears that the predicted temperatures are lower than the values measured during the test. The difference is relatively low: $3.3 \%$. Also, the predicted slope of temperature increase is lower than the experimental one. The consequence is that it remains too large for liquid hydrogen stratification. Note that if there is a difference of $0.1 \mathrm{~K}$ in temperature, it will have a strong impact on the residuals and, therefore, on propellant budget and stage performance. It was then decided to improve the model in several ways. Some of them implied model improvements by implementing a new phase change model and identifying the
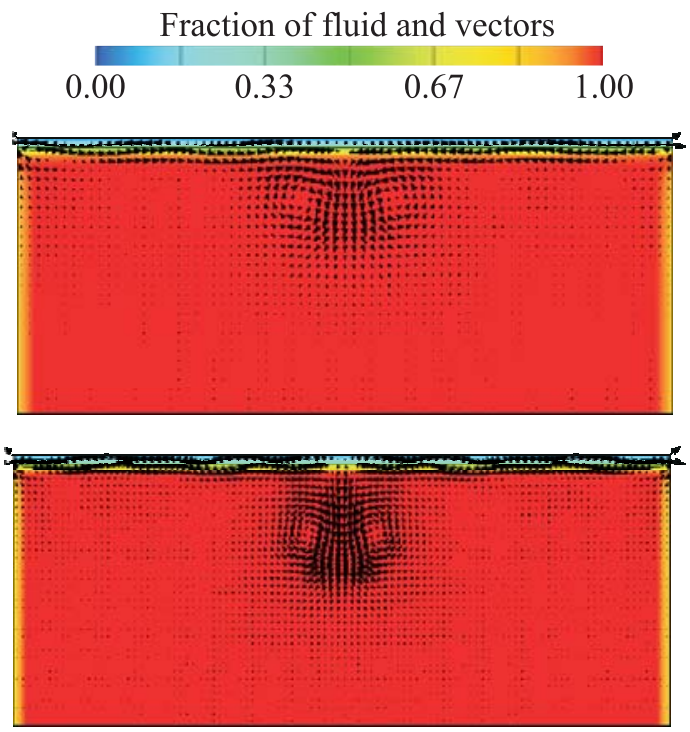

Figure 11 Effect of mesh refinement on the velocity field near the interface. (Refer Dufour, p. 725.)

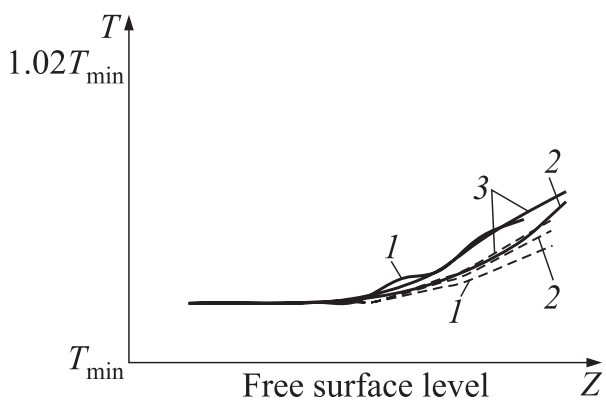

Figure 12 Effect of mesh refinement (solid curves - nominal mesh and dashed curves refined mesh) on the temperature stratification shape: $1-t=50 \mathrm{~s} ; 2-100$; and $3-t$ $=150 \mathrm{~s}$ distribution of thermal fluxes. The other are of purely numerical options like convergence levels and turbulence models.

When the first step is completed, the next step will be to enlarge the physical phenomena modeled with draining and sloshing after the pressurization phases.

The following steps will be to compute a real tank during flight in the boosted phase, and then in the ballistic phase. 


\section{CONCLUDING REMARKS}

The computations performed for experimental configurations have shown that the computer code used allows computing the phenomena encountered in the liquid propellant tanks.

On the one hand, good results were obtained for nonisothermal reorientation. In the microgravity environment at small scale, the results were also promising with low temperature gradient and noncryogenic fluids.

On the other hand, the results obtained for cryogenic fluids were ambiguous. The pressure in the self-pressurization phase was estimated with a good level of accuracy. As for the temperature, the results were is less promising. Due to low temperature of liquid hydrogen, even high accuracy remained insufficient.

Further improvements have to be implemented. Some are linked to numerical and physical models: such as phase change model, use of parallel computation (computation mesh size and speed increase), thermal fluxes, etc. The other are related to industrial cases such as pressurization and sloshing in high gravity conditions, first at intermediate scale (like in this paper), and then at full scale for flight conditions. Works are currently in progress on some of the topics and will be an important point for current and future launchers design and methodology.

\section{ACKNOWLEDGMENTS}

The funding of COMPERE Project in the frame of R\&T CNES is gratefully acknowledged. The author wishes to thanks all people involved in the COMPERE team who contributed with their inputs to the presented studies.

\section{REFERENCES}

1. Dreyer, M., P. Behruzi, C. Colin, A. Dufour, E. Hopfinger, J. Lacapere, B. Legrand, T. Kranz, and J.P. Vila. 2007. Propellant behavior in launcher tanks: An overview of the COMPERE program. 2nd European Conference on Aerospace Sciences.

2. Krahl, R., J. Gertsmann, P. Behruzi, E. Baensch, and M. E. Dreyer. 2008. Dependency of the apparent contact angle on nonisothermal conditions. J. Phys. Fluids 20:042101-1-042101-14.

3. Baumbach, V., E. Hopfinger, and A. Cartellier. 2007. The transient behavior of a large bubble in a vertical tube. J. Fluid Mech. 524:131-42. 